\title{
Analysis of nonlinear vibration energy harvesters using a complex dynamic frequency method
}

\author{
Wang, Z., Wang, W., Zhang, Q., Gu, F. and David Ball, A.,
}

\begin{abstract}
To understand the complicated dynamic behavior of a Nonlinear Piezoelectric Energy Harvester (NPEH), this paper develops an improved Complex Dynamic Frequency (CDF) method based on complex normal form. CDF introduces a dynamic frequency factor and establishes a set of algebraic equations in handling the effect of higher-order nonlinear terms in a wide frequency band to obtain periodic responses of NPEH. Numerical and experimental studies verify that the proposed CDF gives consistent and accurate predictions of the systems with both weak and strong nonlinearity. Furthermore, through an implicit relationship between magnet arrangement and output performance, one may effectively control the sweep frequency with softening and hardening characteristics. That is a major breakthrough toward the further nonlinear design for broad bandwidth harvesters. As the application, the experimental results reveal the high response profiles can be in a wide frequency range from $10.8 \mathrm{~Hz}$ to $24.5 \mathrm{~Hz}$ for the NPEH developed that allows an output power of 9 times higher than the conventional linear structure.
\end{abstract}

Keywords: energy harvester, nonlinear vibration, normal form method, dynamic frequency

\section{Introduction}

The increasing demand for powering microwatt wireless intelligent devices has drawn considerable research attentions towards energy harvesters [1,2], which convert ambient mechanical vibration into electrical energy, realizing the long-lasting energy supply to the devices. The traditional energy harvesters are designed as linear resonators, the shortage of which is that their output power drops dramatically under off-resonance excitations. In order to achieve high-efficiency broadband vibration energy harvesting, researchers have developed kinds of broadband harvesters via nonlinear strategies [3-7].

Herein, the mechanism are realized by using nonlinear magnet force, spring and mechanical constructions. With nonlinear magnetic force and piecewise linearity, a broadband quin-stable energy harvester was introduced by Wang et al. [3]. Leadenham et al. [4] developed an M-shaped nonlinear oscillator that relies on inherent geometric nonlinearity. Pennisi et al. [5] investigated the dynamic behavior of a nonlinear energy harvester in which the magnetic force and the stretching strings interactions produce a cubic stiffness nonlinearity. In [6], a nonlinear energy harvester was proposed that adopts cantilever-surface contact to regulate the bandwidth of the harvester. In another study, Younesian et al. [7] demonstrated that nonlinear spring could be implemented using oblique springs.

Although various nonlinear harvesters have been extensively studied by experiments, calculating the frequency response curves analytically is still a challenging problem. This is because the conventional quantitative analysis methods [8,9] mainly concentrate on weakly nonlinear systems. Among these approaches, the complex normal form (CNF) reduces the workload by introducing complex domain to reduce the order of the weakly nonlinear system. Zhang et al. [10] introduced the undetermined fundamental frequency (UFF) which extends the CNF to the strongly nonlinear system. However, UFF needs multiple iterations to obtain accuracy periodic solutions of the complicated non- $Z_{2}$ symmetry, which increases computational complexity. Zhang et al. [11] presented a dynamic frequency method, where the nonlinearities boil down to a time-period frequency, which greatly decreases the difficulty of quantitative analysis in UFF. However, it has been found that the solution is often less accurate, which leads to inaccurate refinement in tuning the nonlinear harvesters.

This work investigates a complex dynamic frequency method (CDF) to analyze and optimize the harvester with strong nonlinearity to maximize the output power. Following sections will explain the theory of the CDF, numerical verification, and applies the proposed approach to obtain the optimal 
International Journal of Applied Electromagnetics and Mechanics xx (2019) x-x

IOS Press

amplitude-frequency response has been confirmed by the experiments. 


\section{CDF development}

\subsection{CDF theory}

Consider the following general nonlinear system:

$$
\ddot{u}+\omega_{0}^{2} u=f(u, \dot{u}),
$$

where $\omega_{0}$ is the linear frequency, and $f(u, \dot{u})$ can be a power series in terms of displacement $u$ and velocity $\dot{u}$. To improve the UFF, dynamic frequency $\omega(t)$ is introduced and Eq. (1) can be expressed:

$$
u=b+\eta+\bar{\eta}, \quad \dot{u}=i \omega(t)(\eta-\bar{\eta}),
$$

where $\omega(t)=\omega_{10}+\sum_{k=1}^{n} \varepsilon^{k} \omega_{1 k}(t), \quad \varepsilon$ is a bookkeeping parameter, $\omega_{10}$ is the undetermined

fundamental frequency [10], $\omega_{1 k}(t)$ is the the kth order dynamic frequency, and $b$ represents the static equilibrium position. Differentiating Eq. (2) with respect to $t$ yields:

$$
2 \dot{\eta}=\left(\dot{u}-i \ddot{u} \omega(t)^{-1}+i \dot{u} \dot{\omega}(t) \omega(t)^{-2}\right),
$$

where we consider the first order approximation $n=1$. Substituting Eqs. (1) and (2) into Eq. (3), gives:

$$
2 \omega(t)^{2} \eta=-i(\eta-\bar{\eta}) \dot{\omega}(t)+(b+\eta+\bar{\eta}) \omega_{0}^{2}+\omega(t)^{2}(\eta-\bar{\eta})-f\left(b+\eta+\bar{\eta}, i \omega_{10}(\eta-\bar{\eta})\right) .
$$

Applying trigonometric identities to balance the same terms on both sides of the Eq. (4) obtains the unknown variables $a, b, \omega_{10}, \omega_{11}(t)$. No matter how intricate the governing equation is, it will be transformed into four algebraic equations. This will greatly reduce the workload and difficulty of the UFF. Other higher order solution can be determined with the similar procedure. However, the perturbation procedure will be increasingly cumbersome as the order goes up. More importantly, the computational results show that the first order approximation is fairly accurate. The basic algorithm is:

$$
\begin{cases}\text { step 1: balance the constant term } \rightarrow b, & \text { step 2: balance the term of } \cos \omega_{10} t \rightarrow \omega_{10}, \\ \text { step 3: balance the term of } \sin \omega_{10} t \rightarrow a, & \text { step 4: balance the other terms } \rightarrow \omega_{11}(t)\end{cases}
$$

Hence, the approximate solution of Eq. (1) is:

$$
u=b+a \cos \theta(t), \theta(t)=\int \omega(t) d t
$$

It should be specially explained that the integration of $\omega_{11}(t)$ produces a static frequency component $\Lambda_{11}$. Then, as the example, the first order dynamic frequency $\omega=\omega_{10}+\Lambda_{11}$ is applied for the following discussion.

\subsection{Demonstration of $C D F$}

To show the use of CDF proposed, a typical non- $Z_{2}$ symmetric system of Eq. (11) is considered:

$$
\ddot{u}+\omega_{0}^{2} u=\alpha_{2} u^{2}+\alpha_{3} u^{3}+\alpha_{4} u^{4}+\alpha_{5} u^{5}+\beta_{0,1} \dot{u}+\beta_{2,1} u^{2} \dot{u} .
$$

Based on Eqs. (4) and (5), the approximate solution to Eq. (7) can be obtained from the following algebraic equations:

$$
\left\{\begin{aligned}
& \text { step 1: } 15 b\left(\alpha_{3}\left(a^{2}+b^{2}\right)+b \alpha_{2}-\omega_{0}^{2}\right)+3 \alpha_{4}\left(10 a^{2} b^{2}+a^{4}+5 b^{4}\right)+5 a^{2} \alpha_{2}+5 b \alpha_{5}\left(3 a^{2}+b^{2}\right)\left(a^{2}+3 b^{2}\right)=0 \\
& \text { step 2: } \omega_{10}^{2}=\omega_{0}^{2}-\left(a^{2}\left(\alpha_{3}+a^{2} \alpha_{5}\right)+b^{2}\left(3 \alpha_{3}+10 a^{2} \alpha_{5}\right)+b\left(2 \alpha_{2}+4 a^{2} \alpha_{4}+5 \alpha_{5}\right)+4 b^{3} \alpha_{4}\right) \\
& \text { step 3: } a^{2}=-4\left(b^{2}+\beta_{0,1} \beta_{2,1}^{-1}\right) \\
& \text { step 4: } \omega_{11}(t)= 2 a b \beta_{2,1} \sin \omega_{10} t / 3-a / 15 \omega_{10}\left(5 \alpha_{2}+15 b \alpha_{3}+6 a^{2} \alpha_{4}+30 b^{2} \alpha_{4}+30 a^{2} b \alpha_{5}+50 b^{3} \alpha_{5}\right) \cos \omega_{10} t \\
&+4 a^{2}\left(\alpha_{3}+4 b \alpha_{4}+2 a^{2} \alpha_{5}+10 b^{2} \alpha_{5}\right) \sin ^{2} \omega_{10} t / 15 \omega_{10}+a^{3}\left(\alpha_{4}+5 b \alpha_{5}\right) \sin ^{2} \omega_{10} t \cos \omega_{10} t / 5 \omega_{10} \\
&+a^{2} \beta_{2,1} \sin 2 \omega_{10} t / 8-a^{4} \alpha_{5} \sin ^{4} \omega_{10} t / 6 \omega_{10}
\end{aligned}\right.
$$

Fig. 1 presents the phase diagrams of the nonlinear system. It can be seen that the result by CDF is more accurate as it is closer to numerical simulation (NS), compared with that of CNF and UFF for the strongly nonlinear system, shown in G2 plot in the right column. Particularly, Table 1 illustrates that the 
CDF provides excellent approximations to the period and amplitude for both weakly (G1) and strongly (G2) nonlinear systems. This demonstrates that CDF method proposed is more accurate in predicting the behavior of nonlinear systems with different strength of nonlinerity. In future work, focus can be placed on the analyzing partial differential system [12] with the CDF.
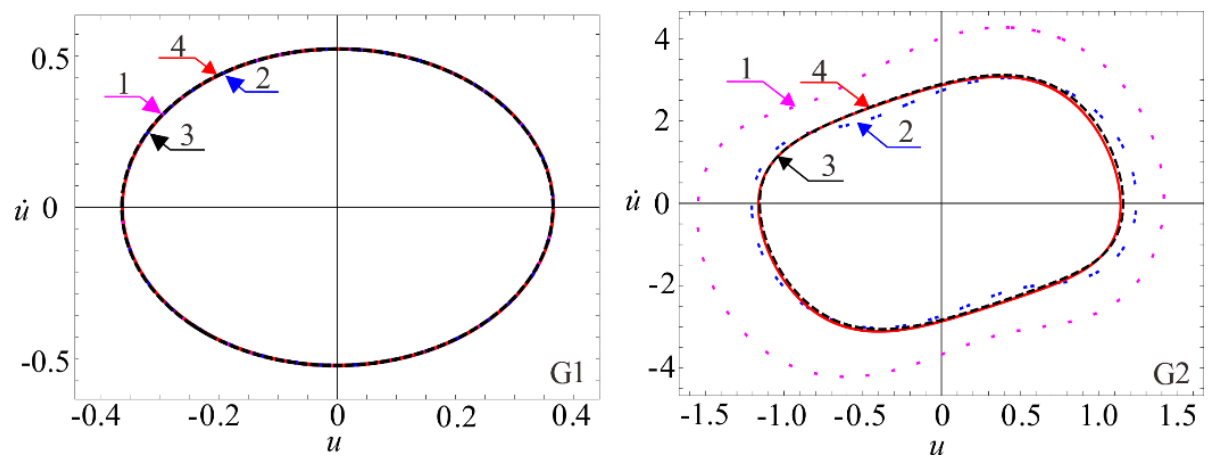

Fig. 1. The phase diagrams of the nonlinear system (1-CNF, 2-UFF, 3-CDF, 4-NS)

$$
\left(\begin{array}{l}
\mathrm{G}_{1}: \omega_{0}=2, \alpha_{2}=0.1, \alpha_{3}=-0.4, \alpha_{4}=-0.1, \alpha_{5}=0.1, \beta_{0,1}=0.01, \beta_{1,2}=-0.3 \\
\mathrm{G}_{2}: \omega_{0}=2, \alpha_{2}=1, \alpha_{3}=-4, \alpha_{4}=-1, \alpha_{5}=1, \beta_{0,1}=1, \beta_{1,2}=-3
\end{array}\right)
$$

Table 1 Comparison of the period and the amplitude of Eq. (7) by different methods

\begin{tabular}{cccccc}
\hline \multirow{2}{*}{ Group } & Variables & NS & CNF & UFF & CDF \\
\hline \multirow{2}{*}{$\mathrm{G}_{1}$} & Period & 3.216 & 3.216 & 3.216 & 3.216 \\
\cline { 2 - 6 } & Amplitude & 0.366 & 0.366 & 0.365 & 0.366 \\
\hline \multirow{2}{*}{$\mathrm{G}_{2}$} & Period & 2.437 & 2.195 & 2.394 & 2.405 \\
\cline { 2 - 6 } & Amplitude & 1.150 & 1.381 & 1.155 & 1.153 \\
\hline
\end{tabular}

\section{Characteristics of the NPEH}

(a)

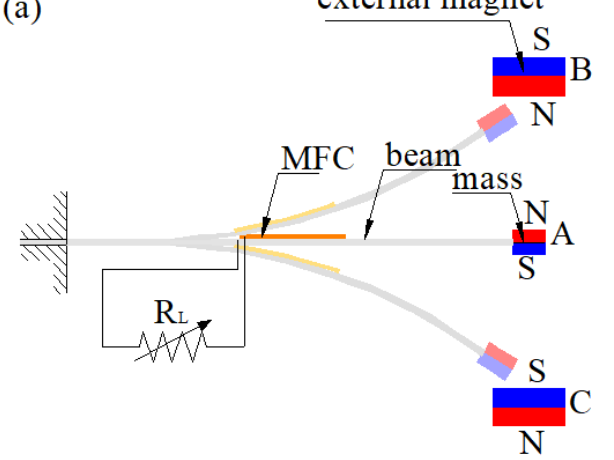

(b)

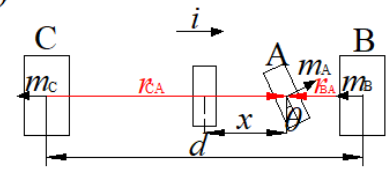

Dimension of NPEH

\begin{tabular}{cc}
\hline beam & $138 \times 15 \times 0.3 \mathrm{~mm}^{3}$ \\
\hline MFC & $85 \times 10 \times 0.3 \mathrm{~mm}^{3}$ \\
\hline A & $15 \times 5 \times 4 \mathrm{~mm}^{3}$ \\
\hline B, C & $20 \times 10 \times 5 \mathrm{~mm}^{3}$ \\
\hline
\end{tabular}

Fig. 2. (a) Schematic diagram of the harvester and (b) configurations of the permanent magnets

As shown in Fig 2, the NPEH system consists of a beryllium bronze cantilever beam, an MFC piezoelectric layer attached to the beam. To amplify the tip displacement of the harvester at a low excitation level, a repulsive magnetic force is introduced into the harvester. Magnet A acts as the proof mass repulsed by the other two fixed magnets B and C. Other parameters of the harvester are in Table2.

Applying the Kirchhoff's and Newton's law, the system with the circuit can be described with [13]:

$$
\left\{\begin{array}{l}
m \ddot{x}+c \dot{x}+k_{1} x+k_{3} x^{3}+k_{5} x^{5}+\chi V=-m \ddot{y} \\
C_{\mathrm{p}} \dot{V}+R_{L}^{-1} V-\chi \dot{x}=0,
\end{array}\right.
$$

where $m, x, c, k_{i}, C_{\mathrm{p}}, R_{\mathrm{L}}, V$ and $\chi$ are mass, relative displacement of the mass, damp, stiffness, capacitance, load resistance, output voltage, and electromechanical coupling coefficient respectively. In particular, the nonlinear stiffness $k_{3}$ and $k_{5}$ are induced by the magnetic force, which will be obtained by simulation with 
the Ansoft Maxwell software. $\ddot{y}=A \cos \Omega \tau$ is the external excitation, where $A$ and $\Omega$ are the amplitude and frequency. To reduce the number of independent parameters, dropping the hats, and considering that $C_{\mathrm{p}}<<1 / R_{\mathrm{L}}$ yields:

$$
\ddot{x}+(2 \xi+\kappa) \dot{x}+\omega_{0}^{2} x+\alpha x^{3}+\beta x^{5}=F \cos (\Omega t),
$$

where $\hat{x}=x / l, t=\omega \tau, \omega_{0}^{2}=k_{1} / m \omega^{2}, \xi=c / 2 m \omega, \alpha=k_{3} l^{2} / m \omega^{2}, \beta=k_{5} l^{4} / m \omega^{2}, \hat{\chi}=\chi^{2} / m \omega^{2} C_{\mathrm{p}}$,

$$
F=-A / l \omega^{2}, \hat{\Omega}=\Omega / \omega, \gamma=1 / C_{\mathrm{p}} R_{L} \omega, \kappa=\chi / \gamma \text {. }
$$

Table 2. Parameter values of the harvester

\begin{tabular}{ccccc}
\hline Parameter & Beam young's modulus & MFC young's modulus & MFC strain coefficient & Capacitance \\
\hline Values & $128 \mathrm{GPa}$ & $33.36 \mathrm{GPa}$ & $-1700 \mathrm{pc} / \mathrm{N}$ & $49.84 \mathrm{nF}$ \\
\hline
\end{tabular}

\subsection{Obtaining steady responses by $C D F$}

To obtain a steady-state response, the response frequency is set to the excitation frequency. Substituting Eq. (2) into Eq. (9), and combining Eq. (5) obtains the amplitude-frequency response function:

$$
a^{2}\left(\Omega^{2}-\omega_{0}^{2}-3 a^{2} \alpha / 4-5 a^{4} \beta / 8\right)^{2}+a^{2} \Omega^{2}(2 \xi+\kappa)^{2}=F^{2}
$$

The power of the load resistance is considered to be the effective power, which can be expressed [13]:

$$
P_{\mathrm{av}}=\int_{0}^{T} \kappa \dot{x}^{2} d t=\kappa \Omega^{2} a^{2} / 2,
$$

where $P_{\text {av }}$ is the averaging power, and $T$ is the time period. The expression of $a^{2}$ can be rewritten as a function of $P_{\text {av }}$ from Eq. (11) and substituted into Eq. (10) results in:

$$
2 P_{\mathrm{av}} / \Omega^{2} \kappa\left(\Omega^{2}-\omega_{0}^{2}-3 \alpha P_{\mathrm{av}} / 2 \Omega^{2} \kappa-5 \beta P_{a v}^{2} / \Omega^{4} \kappa^{2}\right)^{2}+2 P_{\mathrm{av}} \kappa^{-1}(2 \xi+\kappa)^{2}=F^{2} .
$$

\subsection{Dynamic behaviors}

The magnet distance $d$ is one of the key parameters regulating the performance of the harvester. For a smaller magnetic distance, the multi-valued response exists in a broader frequency range. This means that the increasing magnetic distance improves the response amplitude and weakens the possibility of the existence of multiple solutions. Besides, increased magnet distance will increase the size of the prototype and results in reduced energy density. Therefore, appropriate distance should be selected to ensure the conversion efficiency of the harvester. Fig. 3(b) presents that the output of a linear system without the external magnet is much less than that of a nonlinear system under the same excitation condition (linear: $d=\infty, P_{\mathrm{av}}=20.96 \mu \mathrm{W}$; nonlinear: $\left.d=60 \mathrm{~mm}, P_{\mathrm{av}}=167.78 \mu \mathrm{W}\right)$.
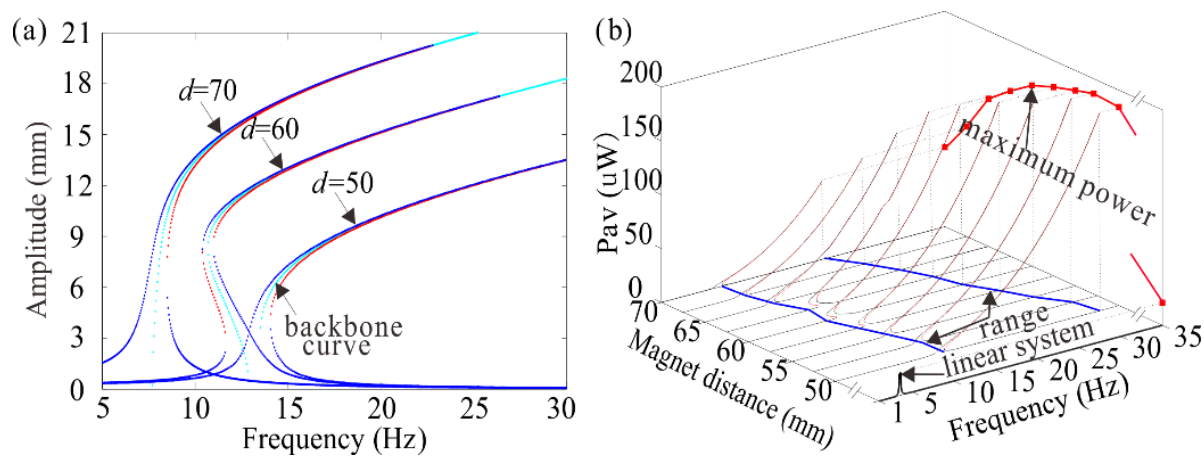

Fig. 3. The influences of magnet distance $d$ on system performances (a) the amplitude-frequency and (b) power-frequency response at the sinusoidal acceleration of $3 \mathrm{~m} / \mathrm{s}^{2}$

In addition, in the magnet distance about $55-65 \mathrm{~mm}$ the harvester has softening and hardening characteristics, which allows harvester to sense ultralow frequency excitation and triggers frequency upconversion to improve the output power. Thus, the effective control of the sweep frequency with softening 
and hardening characteristics will benefit the nonlinear design of the structure.

\section{Experimental validation}

\subsection{Experimental system}

(a)

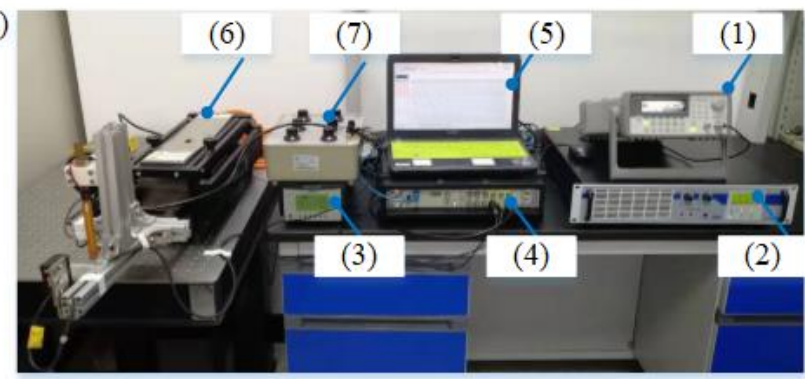

(1) Generator (2) Power amplifier (3) Conditioning amplifier (b)

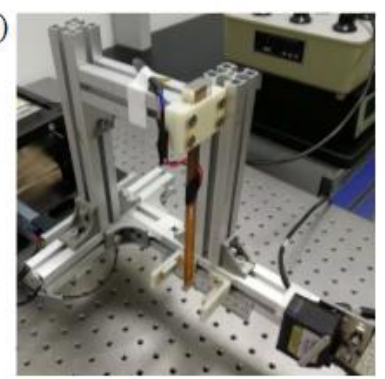

(4) Dynamic signal analyzer

(5) Computer (6) Shaker (7) Resistance box

Fig. 4. (a) Experimental setup and (b) nonlinear energy harvester

The testing system is shown in Fig. 4. The NPEH is excited by an APS 400 shaker, which is driven by signals with different frequency and amplitudes produced by an Agilent 33250A generator and amplified by an APS 125 amplifier. The vibration acceleration and output voltage are measured and recorded by the B\&K 3039 signal analyzer.

\subsection{Results and discussion}

To identify the damping $\xi$ and linear frequency $\omega_{0}$, a small initial excitation was given to the beam [4]. The results indicate that the damping ratio to be determined as 0.0044 and the linear frequency as $81 \mathrm{rad} / \mathrm{s}$.

Fig. 5 plots the root mean square (RMS) voltage and output power of the harvester versus load resistances at the resonance frequency of $22 \mathrm{~Hz}$ under $6 \mathrm{~m} / \mathrm{s}^{2}$ sinusoidal acceleration. Along with the increase of the load resistances, the output voltage increases rapidly and then increases slowly until reaches saturation condition due to the load resistance far surpassing the internal resistance. With the increase of load resistance, the output power rises to the peak value of $184 \mu \mathrm{W}$ and then decreases. The optimum load resistance is $140 \mathrm{k} \Omega$ corresponding to the output peak value.

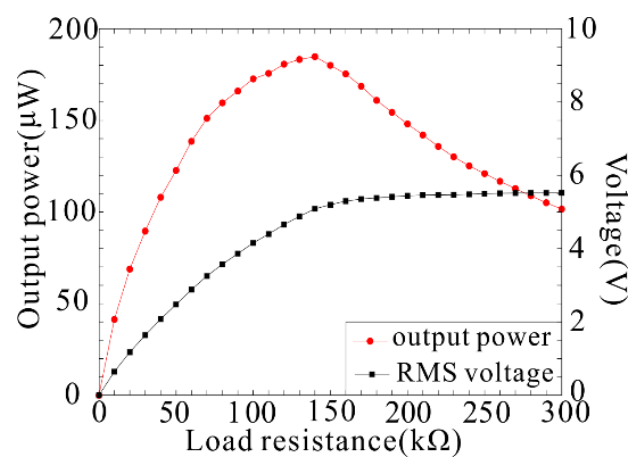

Fig. 5. Output voltage and power versus load resistance at the frequency of $22 \mathrm{~Hz}$ under $6 \mathrm{~m} / \mathrm{s}^{2}$ sinusoidal acceleration

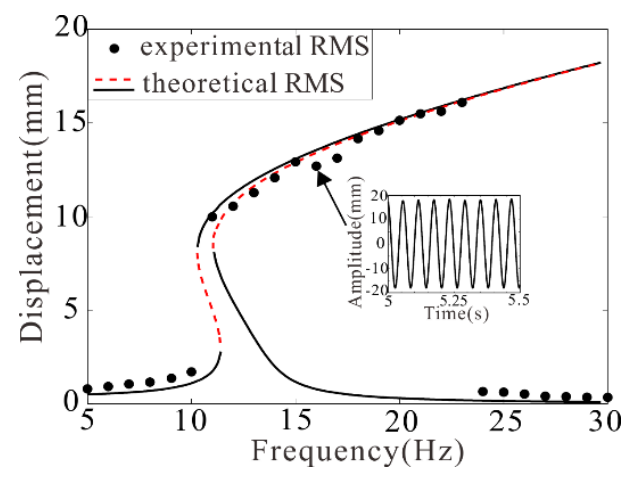

Fig. 6. RMS displacement versus frequency under $6 \mathrm{~m} / \mathrm{s}^{2}$ sinusoidal acceleration

The RMS voltage response of the harvester under acceleration excitation of $6 \mathrm{~m} / \mathrm{s} 2$ is shown in Fig. 6 . It can be seen that the operating frequency band is roughly consistent with the theory in trend, which is predicted in Section 3. This means the proposed approach is able to accurately predict NPEH system with softening and hardening characteristics. The small discrepancy of the frequency band between theory and experiment results is probably due to the simulation of magnetic force, prototype imperfect assembly and unavoidable measuring errors. 


\section{Conclusions}

The complex dynamic frequency method (CDF) captures the high order nonlinear effect into the dynamic frequency to improve the accuracy of analytical computation. It is particularly suitable to analyse and refine the harvester with the complicated nonlinear structures, such as multi-stable, piecewise linearity and nonlinear stiffness and so on. Specifically, it has found that the refined harvester has the potential behaviors allowing for enhanced performances:

a. With the increase of magnet distance, the output power shows the maximum value: $P_{\mathrm{av}}=194.2 \mu \mathrm{W}$ at $d=52.5 \mathrm{~mm}$ and then decreases gradually when the vibration is at the frequency of $22 \mathrm{~Hz}$ with an amplitude of $3 \mathrm{~m} / \mathrm{s}^{2}$. The maximum output power of the nonlinear harvester can achieve more than 9 times, compared with the linear harvester with the similar configuration.

b. The softening characteristic extends the operating band of the harvester to low frequencies, and the hardening characteristic achieves up-frequency to improve the output power and broaden the bandwidth of the harvester.

\section{Acknowledgments}

The work was supported by the National Natural Science Foundation of China (Grant No. 11772218), China-UK NSFC-RS Joint Project (Grant No. 11911530177 in China and IE181496 in UK), Tianjin Research Program of Application Foundation and Advanced Technology (Grant No. 17JCYBJC18900).

\section{References}

[1] X.L. Tang, X.H. Wang, R. Cattley, F.S. Gu and A.D. Ball, Energy harvesting technologies for achieving self-power wireless sensor networks in machine condition monitoring: a review, Sensors-basel. 18(12) (2018), 4113.

[2] G.X. Wang, H. Ding and L.Q. Chen, Dynamic effect of internal resonance caused by gravity on the nonlinear vibration of vertical cantilever beams, J. Sound Vib. 474 (2020), 115265.

[3] C. Wang, Q.C. Zhang and W. Wang, Wideband quin-stable energy harvesting via combined nonlinearity, AIP Adv. 7(4) (2017), 45314

[4] S. Leadenham and A. Erturk, M-shaped asymmetric nonlinear oscillator for broadband vibration energy harvesting: Harmonic balance analysis and experimental validation, J. Sound Vib. 333(23) (2014), 6209-6223.

[5] G. Pennisi, B.P. Mann, N. Naclerio, C. Stephan and G. Michon, Design and experimental study of a nonlinear energy sink coupled to an electromagnetic energy harvester, J. Sound Vib. 437(201) (2018), 340-357.

[6] J.M. Kluger, T.P. Sapsis, and A.H. Slocum, Robust energy harvesting from walking vibrations by means of nonlinear cantilever beams, J. Sound Vib. 341 (2015), 174-194.

[7] D. Younesian and M.R. Alam, Multi-stable mechanisms for high-efficiency and broadband ocean wave energy harvesting, Appl. Energ. 197 (2017), 292-302.

[8] A.H. Nayfeh, The method of normal form, Wiley, 2011.

[9] A.H. Nayfeh, D.T. Mook, Nonlinear oscillations, Wiley, 1995.

[10] Q.C. Zhang, S.Y. Hao and Y.S. Chen, Study on strongly nonlinear vibration systems by normal form theory, J. Vib. Eng. 13(3) (2000), 480-486.

[11] S. Sun and S.Q. Cao, Dynamic modeling and analysis of a bistable piezoelectric cantilever power generation system, Acta Phys. Sin. 61(21) (2012), 210505.

[12] Y.J. Wang, Q.C. Zhang, W. Wang and T.Z. Yang, In-plane dynamics of a fluid-conveying corrugated pipe supported at both ends, Appl. Math. Mech-Engl. 40(8) (2019), 1119-1134.

[13] A. Cammarano, S.A. Neild, S.G. Burrow, D.J. Wang and D.J. Inman, Optimum resistive loads for vibration-based electromagnetic energy harvesters with a stiffening nonlinearity, J. Intel. Mat. Syst. Str. 25(14) (2014), 1757-1770. 